\title{
Predicting a Salient Social Identity from Linguistic Style
}

Miriam Koschate, University of Exeter, UK

Luke Dickens, University College London, UK

Avelie Stuart, University of Exeter, UK

Elahe Naserian, University of Exeter, UK

Alessandra Russo, Imperial College London, UK

Mark Levine, University of Exeter, UK

Word count (incl. abstract, footnotes, references, figures, tables): 9,397

Corresponding author:

Dr Miriam Koschate

Department of Psychology

College of Life and Environmental Sciences

University of Exeter

Washington Singer Laboratories

Perry Road

Exeter

EX4 4QG

United Kingdom

Email: m.koschate-reis@exeter.ac.uk 


\begin{abstract}
Social media data are already being used to classify individuals into mutually exclusive social groups. Here we propose a model based on self-categorization theory that classifies which of two social identities is salient within the same person using text data. Based on over 500,000 online forum posts and seven prototype-based style features, a trained classifier correctly distinguishes between posts written by the same person in two different social contexts $-\mathrm{a}$ parenting forum and a feminist forum - significantly above chance level $(\mathrm{AUC}=.74)$. We then apply the trained classifier to a new dataset $(N=153)$ obtained from an online experiment where salience of group membership is manipulated. We show that our trained model distinguishes between salient parent and feminist identities significantly above chance level when the topic is irrelevant to either identity $(\mathrm{AUC}=.69)$. We discuss applications but also limitations of a text-based prediction of salient social identities.
\end{abstract}

Keywords: social identity salience, computational social science, sociolinguistics, social media, LIWC 


\section{Predicting a Salient Social Identity from Linguistic Style}

Social media and online forums provide millions of users with an opportunity to express themselves. Computerized analyses of text data have provided insights about the psychological self, both in terms of individual differences and group differences. For instance, linguistic style has been used to explore personality differences (Fast \& Funder, 2008; Park et al., 2015; Pennebaker \& King, 1999). In addition to individual differences, linguistic patterns have been examined with regard to various social groups. Several recent studies have reported language differences in online posts between large-scale social groups, such as between men and women (Newman, Groom, Handelman, \& Pennebaker, 2008; Schwartz et al., 2013), Republicans and Democrats (Sylwester \& Purver, 2015), or Christians and Atheists (Ritter, Preston, \& Hernandez, 2014).

Sociolinguistic theories have long held that each of us is part of a large number of different social groups and categories that influence our language use, both in terms of vocabulary and style (e.g., Coupland, 2007; Le Page, Le Page, \& Tabouret-Keller, 1985). The question then arises whether all these groups exert a constant influence on our language use or whether individuals shift their language use in line with the salient social identity. If individuals adjust their language use in predictable ways to the social context, is it then possible to predict which social group membership was salient while a person was writing? Put differently, is it possible to detect different social identities within the same person in different social contexts using only their linguistic style?

In this article, we propose a computerized text analysis method for predicting a salient social identity based on theoretical premises derived from self-categorization theory (SCT; Turner, Hogg, Oakes, Reicher, \& Wetherell, 1987) and sociolinguistic theories. We also provide a case study to (a) demonstrate how such a tool can be created and validated using online data for two specific social identities, and (b) test basic hypotheses derived from SCT. 


\section{Social Identity and Language Use}

The automated analysis of written language has attracted attention from various disciplines, including forensics (e.g., Rashid et al., 2013), political studies (e.g., Laver, Benoit, \& Garry, 2003), and psychology (e.g., Pennebaker \& King, 1999). Computerized text analysis of naturally occurring data from online (e.g. from Facebook, Twitter, chat rooms or blogs) and offline sources (e.g., diaries, manifestos) has allowed researchers to examine how demographic groups, personality traits, and even health conditions can be detected in written language. Here, we focus on a small number of relevant studies where computerized text analysis was used to understand membership in specific social groups or categories:

A growing body of research in psychology examines differences in language use between demographic and social groups using either closed-vocabulary lexicons (Laserna, Seih, \& Pennebaker, 2014; Yarkoni, 2010) such as the program Linguistic Inquiry and Word Count (LIWC; Pennebaker, Mehl, \& Niederhoffer, 2003; Tausczik \& Pennebaker, 2010), or open-vocabulary approaches (e.g., Schwartz et al., 2013). Most of these analyses aim to identify differences in language features between mutually exclusive groups (e.g. men and women, Republicans and Democrats) where an individual usually belongs to one or the other group. To the best of our knowledge, none of these analyses have examined differences in the style of writing of the same person where the person is a member of several intersecting groups.

Similarly, computational approaches using natural language processing (NLP) techniques apply machine learning to accurately classify whether a text is written by a member of one group as opposed to another group (see Nguyen, Doğruöz, Rosé \& de Jong, 2016, for a review). For instance, the ISIS toolkit uses a combination of NLP and authorship attribution to predict age categories (e.g., child/adult) and gender categories (male/female) with remarkably high accuracy (80 \%) using short texts (e.g., from chat rooms; Rashid et al., 
2013). The key difference to psychological research is that computational approaches aim to accurately classify texts to groups rather than merely show significant differences (see Franz \& von Luxburg (2015) for a brief discussion of statistical difference versus classification).

One commonality amongst all these studies is that they assume stability in a person's use of linguistics across different social contexts. Language differences are seen as stable rather than context-dependent. This assumption of stability sits in marked contrast to sociolinguistic theories, such as accommodation theory and speaker design theory, and social psychological theories, particularly SCT.

\section{Linguistic Style Variation}

Early theorists in sociolinguistics such as Labov (1968/2006) suggested a social dimension to intra-individual language use. In particular, he suggested that social variables would affect stylistic choices. Early sociolinguistic models focused on the formality of the eliciting task, and the ways in which linguistic style was associated with social stratification. In contrast, later models and theories started to examine how communication partners affect linguistic style and the role groups play in this.

The most extreme form of intra-individual linguistic variation can be seen in code switching. Code switching is defined as "alternations of linguistic varieties within the same conversation" (Myers-Scotton, 2003, p. 1). This can take many forms, from switching from one language to another within the same sentence to moving from a formal to an informal style during a conversation with a stranger.

Accommodation theory, for instance, suggests that partners in a conversation adjust their language to each other and that such accommodation is perceived positively by conversation partners (Giles, Taylor, \& Bourhis, 1973). Here, linguistic style variation is thought to stem directly from style differences between conversational partners and attempts to adjust to them. Such stylistic convergence with conversational partners may also explain 
homogeneity of styles within social groups. For instance, a study by Tamburrini, Cinnirella, Jansen and Bryden (2015) examined language style convergence on Twitter. They found that messages sent internally (within a Twitter community) are linguistically more similar than messages sent externally. One problematic aspect of accommodation theory, however, is that it assumes an active conversational engagement amongst all individuals within a group. While this is realistic for small-scale face-to-face groups and even dispersed groups interacting through social media (e.g., Twitter communities or Facebook groups), such a mechanism appears unlikely to create common language use in very large-scale social categories (e.g., parents, religious communities, political groups).

Audience design theory (Bell, 1984) shares similarities with accommodation theory in that it also sees the conversation partner as the source of variation. However, here the adjustment is not towards an individual conversational partner but towards an audience in the form of a social group and their (assumed) linguistic norms. Similar to accommodation theory, it suggests a responsive shift where the author or speaker designs their style for a particular audience to win their approval. In addition, audience design theory also proposes an initiative dimension, where the author uses the style of a group that is absent from the conversation for its associated prestige. Hence, audience design theory suggests differences in stylistic norms between groups as the source of stylistic variation. However, it does not focus on the group membership of the author but rather on that of the audience.

In contrast, speaker design theory points towards the topic as a source of stylistic variation. For instance, Estes-Schilling (1988) found that school friends would use a similar style when talking about shared experiences but would diverge stylistically when the topic of conversation shifted towards their different ethnic groups. Similarly, Coupland (1980) found that travel agents did not only shift their style from one customer to the next (in line with 
accommodation theory) but that style shifts occurred between work-related topics and other topics.

\section{Self-Categorization Theory (SCT)}

SCT suggests that the social context determines which group norms we are following in any given situation (Turner et al., 1987). More specifically, cues in the social context determine which social identity is salient out of all the group memberships that we inhabit. This identity then affects our behavior through associated group norms. Audience and conversational topic are two out of several social cues that determine social identity salience (e.g., Barreto, Spears, Ellemers, \& Shahinper, 2003; Yopyk \& Prentice, 2005), alongside factors such as group size (e.g., Mullen, 1991) and emotions (e.g., Livingstone, Spears, Manstead, Bruder, \& Shepherd, 2011). Importantly, SCT proposes that social identities, including demographic categories, exert a dynamic rather than constant influence on our behavior. This context-dependent nature of identity salience translates into a dynamic shift in behavior. Several studies have shown that identity salience affects attitudes and behavior (e.g., Haslam, Oakes, Reynolds, \& Turner, 1999; Hogg \& Terry, 2000; Shih, Pittinsky \& Ambady, 1999). However, dynamic shifts in behavior within the same individual are rarely examined.

Individuals are also active users and communicators of their social identities (Klein, Spears, \& Reicher, 2007). They strive to communicate a desired social identity to others by behaving in line with group norms: both towards the in-group to assert their group membership and towards outgroup members to achieve intergroup differentiation. In sum, SCT suggests that linguistic style shifts systematically with the social context inasmuch as the context affects the salience of social identities held by the individual.

If social identity is indeed linked to systematic language style shifts, then an agreement on stylistic features within groups is necessary. Group members are generally motivated to 
achieve consensus on characteristics of the group - the group prototype (Haslam et al., 1999; Hogg \& Reid, 2006). In turn, group normative behavior can be deduced from the group prototype (Turner, 1991). Unlike a group stereotype which is a broader societal view of the group, a prototype reflects how the group see themselves. We propose that linguistic style differences stem in large part - although not exclusively - from group prototypes, and can therefore be predicted from knowledge of group prototypes.

In the following, we will examine whether differences in the prototype of two social groups in terms of linguistic style can be used to predict which of two social identities is salient within the same person holding both social identities. Our research adds to the current literature in three important ways: First, we will test whether the salience of different identities can be detected in short texts written by the same person holding both identities (social identity shifts) rather than by different members of mutually exclusive groups. Second, we will examine whether linguistic style is affected by the group prototype of the salient social group (prototype-based linguistic style) to provide insights into whether social identities play a role in intra-individual style shifts. Third, we will assess whether a salient social identity can be detected when potential confounds of linguistic style shifts such as topic, audience, and individual differences are controlled for.

Based on SCT, we expect the pattern of stylistic features of the two groups to be sufficiently distinct that it is possible to accurately classify from which group a text stems (Hypothesis 1). More specifically, we expect that the two groups are sufficiently distinct in the use of a small number of prototype-based features that it is possible to accurately classify from which group a text stems (Hypothesis 2). We also expect that the trained classifiers will reliably distinguish between the two social identities within a single person who is a member of both social groups (Hypothesis 3).

\section{Current Research}


In order to test our predictions based on SCT, we chose two social groups that show a good overlap in membership but distinctiveness in their prototype. This allows us to test for between-group differences as well as within-person shifts in language style. Parents and feminists are two such distinct, large-scale social groups with a reasonably good overlap in group membership and a good online presence.

First, we will use online forum posts to train and cross-validate a linear logistic regression model that classifies which of the two social identities was salient when writing a post (Study 1). This trained model will then be used to distinguish between identities in a test set of data that was obtained in an online experiment where social identity salience was manipulated, and where topic, audience, and individual differences including demographic differences were controlled for (Study 2).

\section{Study 1}

Study 1 consists of three separate steps to build our classifier: First, we will quantify stylistic features based on online forum posts using the closed-vocabulary method LIWC. Second, we will train the classifier on posts from two different forums of the same online platform that represent the two groups of interest using prototype-based features, and comparing to an all-features model. Third, we will test the classifier on posts written by the same person in the two different forums to test for intra-individual social identity shifts.

\section{Method}

Online data. The discussion forum data were gathered from the online website Mumsnet (www.mumsnet.com/talk), the largest parent online network in the UK, with the kind permission of Mumsnet. This site provides different sub-forums, in which users can discuss particular topics and themes. We analyzed posts from two sub-forums, 'Being a Parent' and 'Feminism'. The posts were collected in September 2012 from 2,500 threads per sub-forum. Every person who wishes to contribute to Mumsnet is required to create a user 
account with a unique username. Hence, posts from the same author can be matched by the username, irrespective of the sub-forum in which they were posted.

Overall, our sample consists of $N=638,226$ posts written by $N=19,816$ different users. A total of $n=402,394$ posts from $n=12,733$ users were collected from the 'Being a Parent' sub-forum and $n=235,832$ posts from $n=9,985$ users from the 'Feminism' subforum, with $n=2,902$ users having posted in both forums. Although it is not possible to extract demographic data, Pedersen and Smithson (2013) found in their study of Mumsnet users that the majority of users are mothers (97\%), between 31-40 years old (61\%), with a high education level (34\% have a university degree).

Preparation of data. We excluded "posts" that only included an administrative message from Mumsnet rather than a genuine message by the user (e.g., "Message withdrawn" or "Message deleted by Mumsnet"). All resulting LIWC features were normalized using z-standardization.

Training and test samples. We used bootstrapping to train our model 20 times on subsets of 100,000 posts. For each subset, half of the posts were randomly sampled from the full set of 'Being a Parent' posts and the other half were randomly sampled from the full set of 'Feminism' posts. In the test stage, the trained classifier was cross-validated 20 times on posts from a random sample of $n=1,000$ users who had posted in both forums, using one random post from each of the two forums for each user. ${ }^{1}$

Feature extraction. For feature extraction from texts, we used the Linguistic Inquiry and Word Count (LIWC) software (Pennebaker, Booth, \& Francis, 2007) to quantify linguistic markers. LIWC is a widely-used expert based system that maps each word to one or more linguistic features so that documents are represented as a normalized frequency of each feature. LIWC mappings have been developed and refined over a number of years by panels 
of researchers in psychology and language, and are based on a variety of corpora (Pennebaker, Chung, Ireland, Gonzales, \& Booth, 2007).

LIWC provides counts for 'part-of-speech categories' and 'context-specific categories' (Schwartz et al., 2013). Part-of-speech categories represent words used generally across multiple contexts such as different grammatical categories (e.g., different types of pronouns) and basic psychological categories (e.g., time words, positive/negative emotion words). We use these categories as style features in our studies. Overall, 49 different non-redundant style features can be extracted from each text. In line with sociolinguistic theory, "context-specific categories' that reflect the content or topic of conversation (e.g., family, work) will not be used. This also reduces the risk of producing somewhat trivial results based on the differing content of group conversations.

Selection of features based on prototypes. The prototypes of parents and feminists differ in a number of aspects: Based on the literature, characteristics that describe feminists endorsed by those seeing themselves as feminists are intelligent, assertive, political, and liberal (Roy, Weibust, \& Miller, 2007; Twenge \& Zucker, 1999). In contrast, parenthood is characterized as being loving, warm, caring, generous and protective (Ganong \& Coleman, 1995) as well as child-centered and emotionally involved (Ennis, 2014; Hays, 1998).

Although initially seen as a stereotype (Ganong \& Coleman, 1995), these characteristics form part of the ideology of 'intensive mothering' predominant among parents in the US and other Western countries (Ennis, 2014; Hays, 1998). A recent study by Ishizuka (2018) shows that the majority of US parents have internalized the ideology of 'intensive parenting' and 'childcentered parenting', suggesting that these characteristics have become part of the parenting prototype.

These different prototypes lead us to predict that users whose feminist rather than parent identity is salient are more likely to: write in a style that is intellectual, e.g. using more 
long words ('Sixltr' in LIWC), avoid gendered language, e.g. by using the third person singular pronoun ('they'), and use more negative emotion words ('negemo'). In contrast, individuals whose parent rather than feminist identity is salient will tend to use language that: centers around specific others by using third person singular ('shehe'), expresses the concrete rather than the abstract by using 'time' words such as yesterday/last week, is inclusive by using words such as and/with/together ('incl'), and reflects positive emotions ('posemo'). We will use these seven prototype-based features to build our classifier.

Training the classifier. We begin by examining whether the language style used for posts in a forum for parents can be distinguished from that used for posts in a forum for feminists on the same online platform. The criterion for our logistic regression models will therefore be the forum from which the post originates.

Posts from users who have only posted in one of the two forums, along with posts from those who have used both forums will be included in the training dataset. This 'betweenforums' design is used to allow for the widest possible sample of parents and feminists on the platform to be included in the training of the model.

As part of model training, we identify those features that are most predictive of differences between forums: first, by simply including all stylistic features within the model, and allowing the fitting procedure to ignore non-informative features; second, by using a small selection of prototype-based stylistic features. This procedure allows us to compare whether a theoretically derived model using prototypical features is valid or whether relevant information would be lost.

We evaluate our models first by drawing on posts irrespective of their length, then only including longer posts with $Q 1=25$ words or more for both training and testing. This restriction represents a trade-off between excluding very short posts with insufficient information for any reliable prediction, and ensuring the test set can be made sufficiently 
large for a robust evaluation (see Supplementary Information for AUCs under different cutoff points).

Testing the classifier. Next, we test the model on its ability to differentiate between the two forums on a separate subset of posts originating from users who have contributed to both forums. The 'within-user' design of the test dataset allows for a more stringent test of the theory by excluding stable individual differences between the two social groups such as demographic differences (e.g., social class, level of education) and personality differences. It also allows us to examine whether systematic social identity shifts occur within the same person when the social context changes (i.e., the person contributes to forums reflective of different social identities).

\section{Results}

Training. In the first step, we trained the classifier on posts randomly sampled from all users in the overall sample. Without length restrictions, and using all available style features, prediction accuracy was high with mean AUC $=.86$ (see Figure 1 for AUCs and $95 \%$ confidence intervals for all test and training models). By restricting posts to those of 25 words or more, prediction accuracy increased to mean $\mathrm{AUC}=.93$. These results support $\mathrm{H} 1$ that the pattern of stylistic features of the two groups is sufficiently distinct that it is possible to accurately classify from which group a text stems.

In the next step, we restricted the style features to the seven features that reflect differences between the two group prototypes. Prediction accuracy remained comparable with mean $\mathrm{AUC}=.81$ without length restrictions, and mean $\mathrm{AUC}=.88$ when restricting posts to those with 25 words or more. Results show that using a small number of prototypebased features produces results that are only marginally smaller than when all 49 available style features are used. Notably, the seven prototype-based features are part of the full set of 
49 style features. This finding supports $\mathrm{H} 2$ that a prototype-based model with only a small number of features produces prediction accuracies above chance level.

Testing. To validate our models, we tested them on posts by users who had posted in both forums, that is, those forum users that are members of both the parent and feminist group. Prediction accuracy was well above chance level of .50, irrespective of feature selection or length restrictions (see Figure 1): Using all style features and posts without length restrictions produced a prediction accuracy of $\mathrm{AUC}=.71$, and improved to $\mathrm{AUC}=.76$ when only longer posts were considered. Similarly, using only prototype-based features produced a predictive accuracy of $\mathrm{AUC}=.70$ for unrestricted post length and $\mathrm{AUC}=.74$ for longer posts.

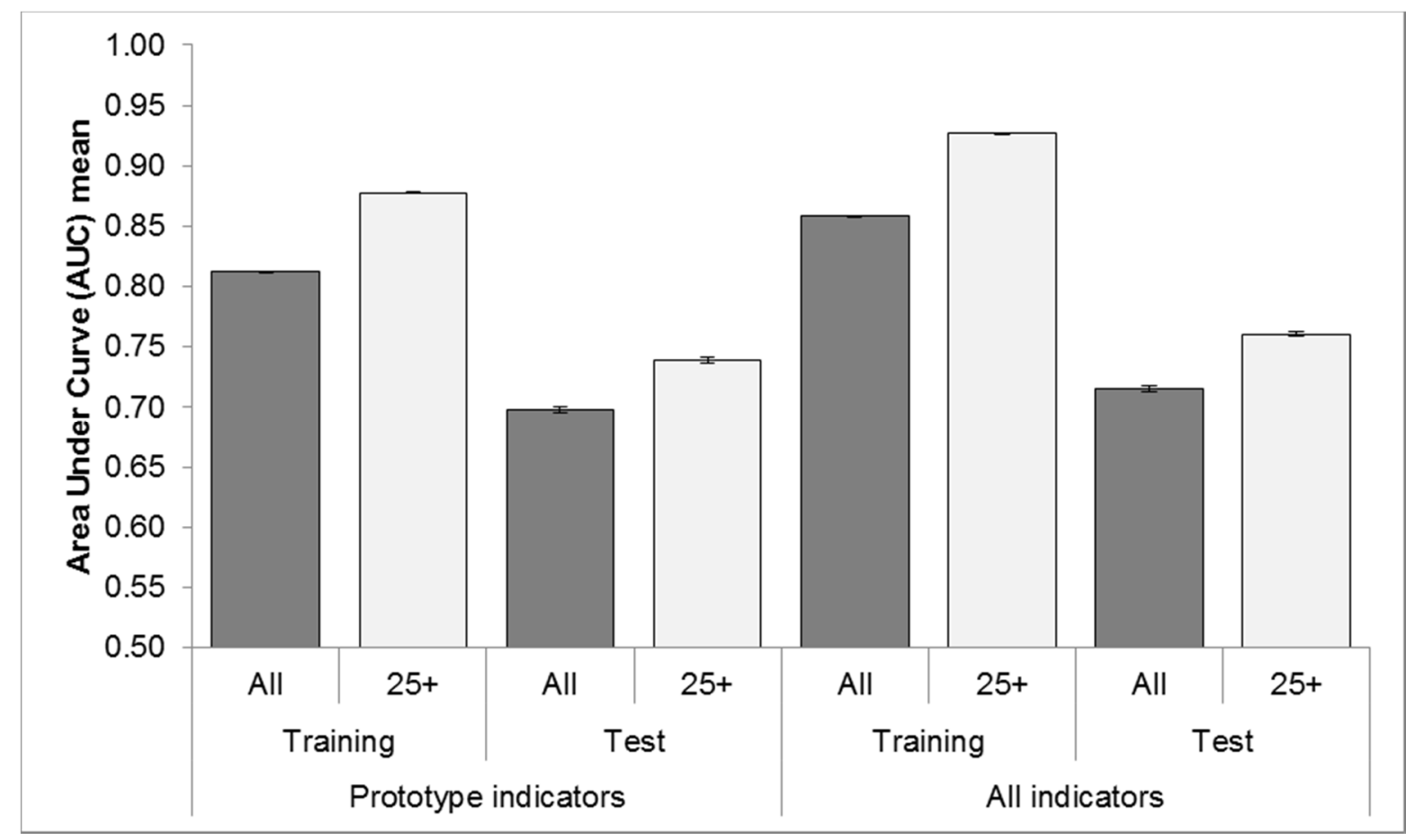

Figure 1. Predictive performance (mean AUC) for Study 1 training and test data with error bars showing $95 \%$ confidence intervals.

These results demonstrate that, even though there is additional information present in these other style features, the vast majority of information is captured by our seven prototype- 
based features, supporting H2. Importantly, results also support H3 by showing that posts by the same user can be accurately classified, indicating intra-individual style shifts in line with changes in social identity salience.

Importantly, the test sample of individuals who had posted in both forums controls for stable individual differences between the two social groups such as age, education and personality differences. Figure 2 illustrates the extent of this shift for a single user.

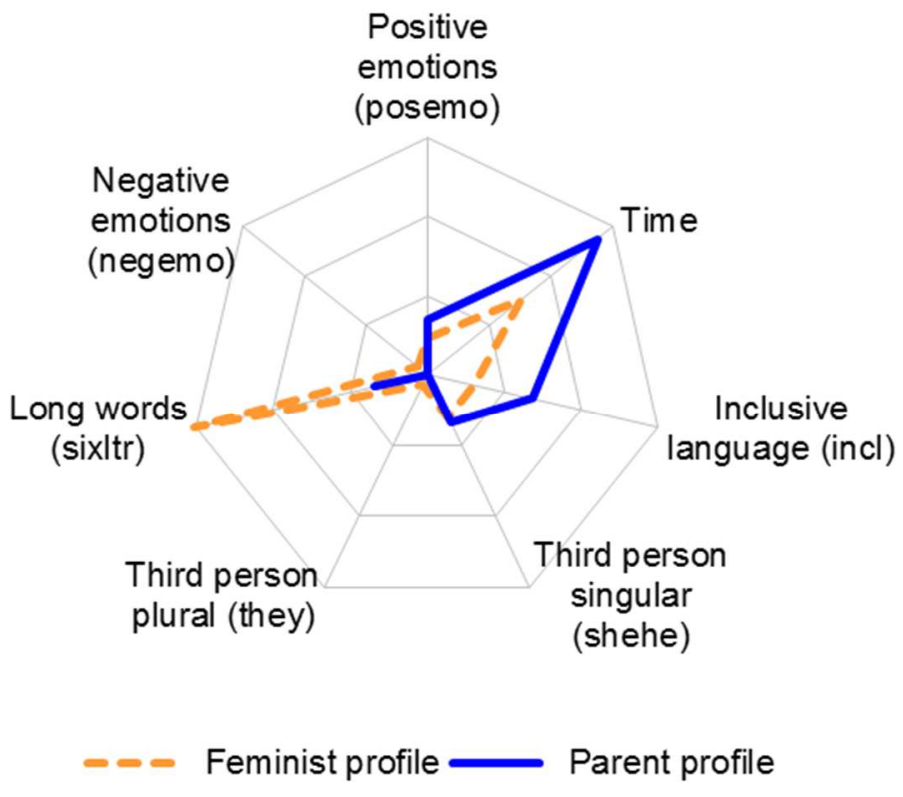

Figure 2. Intra-individual style shift of a single user on prototype-based features

To further investigate the relevance and uniqueness of the prototype-based features, we trained a separate logistic regression model on each of the stylistic LIWC features individually (with the same training and testing procedure). Figure 3 shows the predictive performance for each feature individually. Findings indicate that prototype-based features are 


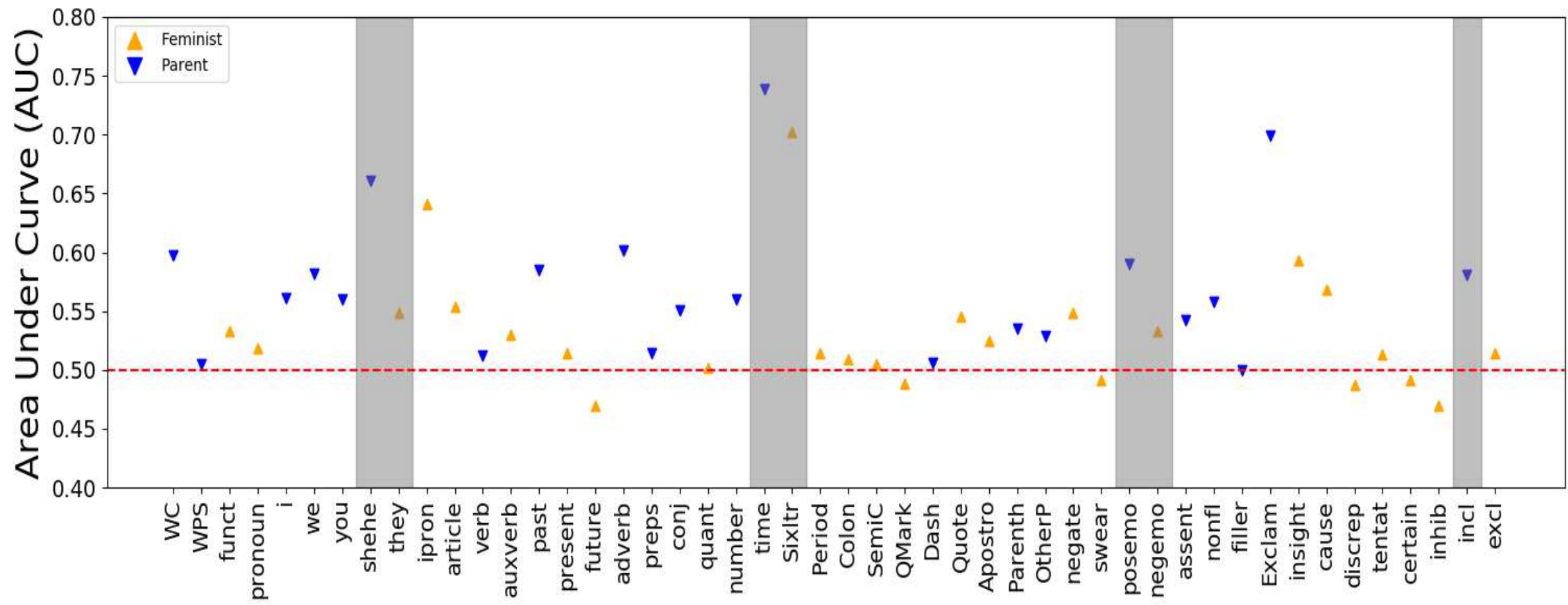

Figure 3. Individual predictive performance (AUC) of all 49 LIWC features ( $\nabla$ indicate positive predictors of a parent identity; $\Delta$ indicate positive predictors of a feminist identity; grey bars indicate features that were selected as reflecting differences in prototype). 
among the best performing individual predictors, but also that they are not unique in being able to differentiate between forums.

\section{Discussion}

Our study demonstrates that linguistic style differs between forums associated with two different but intersecting social identities. Differences in linguistic style were pronounced enough to allow us to predict which forum a post had come from with good accuracy. A small number of prototype-based features provided enough information to classify the salient identity of an individual well above chance level. This suggests that our literature-based prediction of the prototype was reflective of the linguistic norms of the two identities rather than being based on a stereotype (i.e., incorrect generalizations about the two groups). It also provides first evidence that intra-individual style shifts are linked to a salient social identity.

Furthermore, our cross-validation on a within-participant sample (i.e., where participants had posted in both forums) shows that differences in style are not simply a reflection of the different demographic composition of the groups. It also suggests that individuals shift their linguistic style from one forum to the next, that is, linguistic style is not intra-individually stable but dynamic and context-dependent, in line with SCT and sociolinguistic theories. However, the forum data alone can only serve as a first indication that social identity salience produces these style shifts. For instance, heterogeneity of style within forums may simply stem from accommodation to the norms of the community rather than a salient social identity. In Study 2, we will test the reliability of the prototype-based classifier, which we trained and validated in Study 1, by applying it to a new dataset where we manipulate the salience of the social identity in an experiment.

In addition, by restricting ourselves to style features rather than all features provided by LIWC, we are aiming for a model that is less prone to influence from the topic of conversation, thus avoiding potentially trivial results (e.g., parents use more words related to 
'family' than feminists). However, style features including grammar are not independent of topic and associated semantics (Goldberg, 1995). Using online data means that we cannot control for conversational topic. In contrast, in Study 2, we can experimentally control which topics people write about, and whether the topic is identity-relevant or not.

Using experimental data to validate our model also provides us with an opportunity to directly test our hypothesis that intra-individual style differences are due to differences in the salient social identity. Rather than having to use forums as proxies for identities, we can assess identities through self-report and manipulate the salience of identities for those that hold both relevant identities. While it is likely that the majority of users posting in Mumsnet forums hold the respective social identity, and that the context of the forum would make the relevant identity salient, this cannot be easily ascertained.

Furthermore, an experimental validation allows us to test how widely our model generalizes, that is, whether it can predict social identity for participants that have not used the platform on which the model was trained. In Study 1, using the same platform (i.e., Mumsnet website) gave us the opportunity to track users across forums, and thus test our classifier on posts from different forums by the same group of people. In Study 2, we will recruit from several different online sources to test how widely our model generalizes to parents and feminists with different demographics to Mumsnet users.

\section{Study 2}

In Study 2, we will use the classifiers trained in Study 1 on a new dataset from an online experiment. The experiment allows us to use self-reported social identities as the prediction criterion, rather than the proxy 'forum' as in Study 1. Moreover, for those holding both social identities, salience of identity can be manipulated in order to test whether our model can, indeed, predict which social identity was salient during writing. The experiment also allows us to control for conversational topic, exclude variation in audience as the source 
of differences in style, and control for demographic and other individual differences. We will recruit participants from other websites than Mumsnet to test whether the classifier trained on Mumsnet generalizes to other contexts and demographics.

\section{Method}

We collected data in an online experiment that simulates the format of a forum entry while at the same time providing us with additional information on participants' identity and the opportunity to systematically vary which identity is cognitively salient and which topic is being written about.

Participants and design. We calculated the target sample size (total $N=32$; (Goksuluk, Kormaz, Zararsiz, \& Karaagaoglu, 2016) for testing our prototype-based classifier from Study 1 with power of .80 and an AUC of .74 (see Study 1), assuming an equal ratio of parents and feminists. We over-recruited for the salience conditions (i.e., where participants self-report as being a parent and feminist) to ensure that sufficient numbers of native English speakers were included in the sample and dropout would not pose a problem. We expected that this strategy would produce larger than required participant numbers for the single identity conditions (i.e., where participants self-report as being either a parent or feminist). Participants were recruited via advertising in forums such as Netmums UK (an alternative platform to Mumsnet), Reddit (r/feminism), Facebook, Twitter, and through a paid online recruitment platform in the UK, Prolific Academic. Notably, the study was not advertised on Mumsnet, and only $n=8$ participants (5\%) indicated that they used Mumsnet.

A total of $N=177$ native English speakers participated in the online study. Twenty-four participants who indicated that they were neither a parent nor a feminist were excluded from analysis, leaving a total of $N=153$. The majority of participants were female $(n=116,76$ $\%), n=34(22 \%)$ were male, and $n=3(2 \%)$ had another gender status. Participants were between 18 and 69 years old $(M=35.31, S D=11.43)$ and had between 0 and 4 children $(M=$ 
$1.67, S D=1.17)$. The majority of participants reported to be employees ( $59 \%$; full-time, part-time, self-employed, and temporary), $18 \%$ said they were in education, and $18 \%$ that they were currently at home (stay-at-home, retired, or unemployed), with $4 \%$ not reporting their current employment status. Participants live in various regions of the UK, with 62 of 83 UK counties plus London represented in our sample.

The study follows a 2 (identity: parent $\mathrm{v}$ feminist) x 2 (salience: single identity v salient identity) x 3 (topic: parent, feminist, identity-irrelevant) design, with identity and salience as between-participants factors and topic as a within-participants factor. Participants indicated at the beginning of the study whether they consider themselves to be a parent (yes/no) and/or a feminist (yes/no). Participants who indicated that they were both a parent and feminist were randomized to one of the two salient identity conditions: salient identity parent: $n=21$; salient identity feminist: $n=22$. Participants who indicated only one of the two identities make up the single identity parent condition $(n=39)$ and the single identity feminist condition $(n=71)$, respectively.

Social identity salience manipulation. We randomly assigned those who had indicated both identities to either a feminist or parent salience condition. In these conditions, participants were asked to think of themselves either as a feminist or a parent, respectively. Furthermore, they were asked to write down "up to three things that you and other [feminists/parents] do..." (a) often, (b) rarely, (c) well, and (d) badly (Haslam, Turner, Oakes, McGarty, \& Reynolds, 1997). This identity salience manipulation psychologically activates the respective identity by focusing participants on both positive and negative similarities with other group members and the group prototype without introducing a threat from an outgroup (Haslam, 2004). 
Topic. Every participant was asked to write three to five sentences (roughly 25 words) addressing each of three pre-defined topics: healthy mealtimes (parent topic), objectification of women (feminist topic), and climate change (identity-irrelevant topic).

The three topics were chosen based on a pre-test. In the pre-test, $N=13$ participants $(9$ women (69\%) and 4 men, age: $18-49$ years, $M=27.77, S D=17.86)$ rated 26 topics on whether the topic is typical for a conversation among feminists and parents, respectively. We selected a topic that was perceived to be typical for feminists but not parents (objectification of women: within-participant t-test, $t(10)=4.03, p=.002$ ), a topic that was perceived to be typical for parents but not feminists (healthy mealtimes: $t(11)=6.20, p<.001$ ), and a topic that was perceived as being neither typical for conversations among parents nor feminists (climate change, $t(11)=0.00, p=1.00)$.

Audience. The audience was held constant across conditions by providing participants at the beginning of the study with information that any texts the participant writes would only be seen by the researchers, and not by any other person. No other information about the researchers was provided.

Demographic measures. We asked participants to indicate their age in years (open answer), their gender (male, female, other status), their employment status, the number of own children, whether they are a native speaker of English (yes/no), and their UK county of residence.

Regression weights. In order to calculate the probability of an individual having a feminist rather than a parent identity salient, we used the regression weights for the following seven style features based on the prototype-based model that uses longer posts ( 25 words or more) from Study 1: 'Sixltr' $(b=0.16)$, 'they' $(b=0.19)$, 'shehe' $(b=-0.13)$, 'incl' $(b=-$ $0.08)$, 'time' $(b=-0.30)$, 'posemo' $(b=-0.12)$, and 'negemo' $(b=0.06)$. 
Procedure. The study was run on the online survey software LimeSurvey (Schmitz, 2012). Participants were first presented with an information sheet that briefly outlined the study, data protection (including 'audience' information) and other ethically relevant information to ensure informed consent. After providing their consent, participants were first asked whether they consider themselves to be a parent/feminist, this was followed by other demographic questions. Those with both identities were then randomized to one of the two salient identity conditions and received the identity salience manipulation. All participants were then asked to write short paragraphs on the three topics. Participants were debriefed and thanked for their participation.

\section{Results}

Preliminary analyses. First, we examined differences between parents and feminists in a number of variables to understand whether the two groups differ from each other in relevant aspects and check whether randomization for the salient identity conditions was successful. Results indicate that feminists in the single identity condition, that is, those who said they are a feminist but not a parent, differ from the three other conditions with regards to several variables: they do not have children $\left(F(3,96)=33.52, p<.001, \eta^{2}{ }_{p}=.512\right)$, are younger than participants in the other conditions $\left(F(3,96)=12.13, p<.001, \eta^{2}=.275\right)$, and are more likely to be in education $\left(\mathrm{X}^{2}(3, N=147)=18.01, p<.001\right)$ than participants from any of the other three groups. There were no significant gender differences between the parent single identity (13 men, 25 women) and feminist single identity conditions (19 men, 50 women), $\mathrm{X}^{2}(1, N=$ $107)=0.52, p=.471$. The two salient identity conditions featured only one male participant each $\left(\mathrm{X}^{2}(1, N=43)=0.001, p=.973\right.$. Furthermore, participants in the salience conditions did not differ significantly from each other in age $(t(41)=0.71, p=.479)$, number of children $(t(41)=0.52, p=.608)$ or employment status $\left(\mathrm{X}^{2}(2, N=40)=0.76, p=.685\right)$, suggesting that randomization was successful. 
Classification results. Using the prototype-based classifier trained on online forum posts of 25 words or more in Study 1, we calculated the probabilities of participants writing as a feminist or parent, respectively, using the relevant LIWC features extracted from the three texts (for full results see Table 1).

Table 1

Predictive performance by condition, topic and sample for Study 2

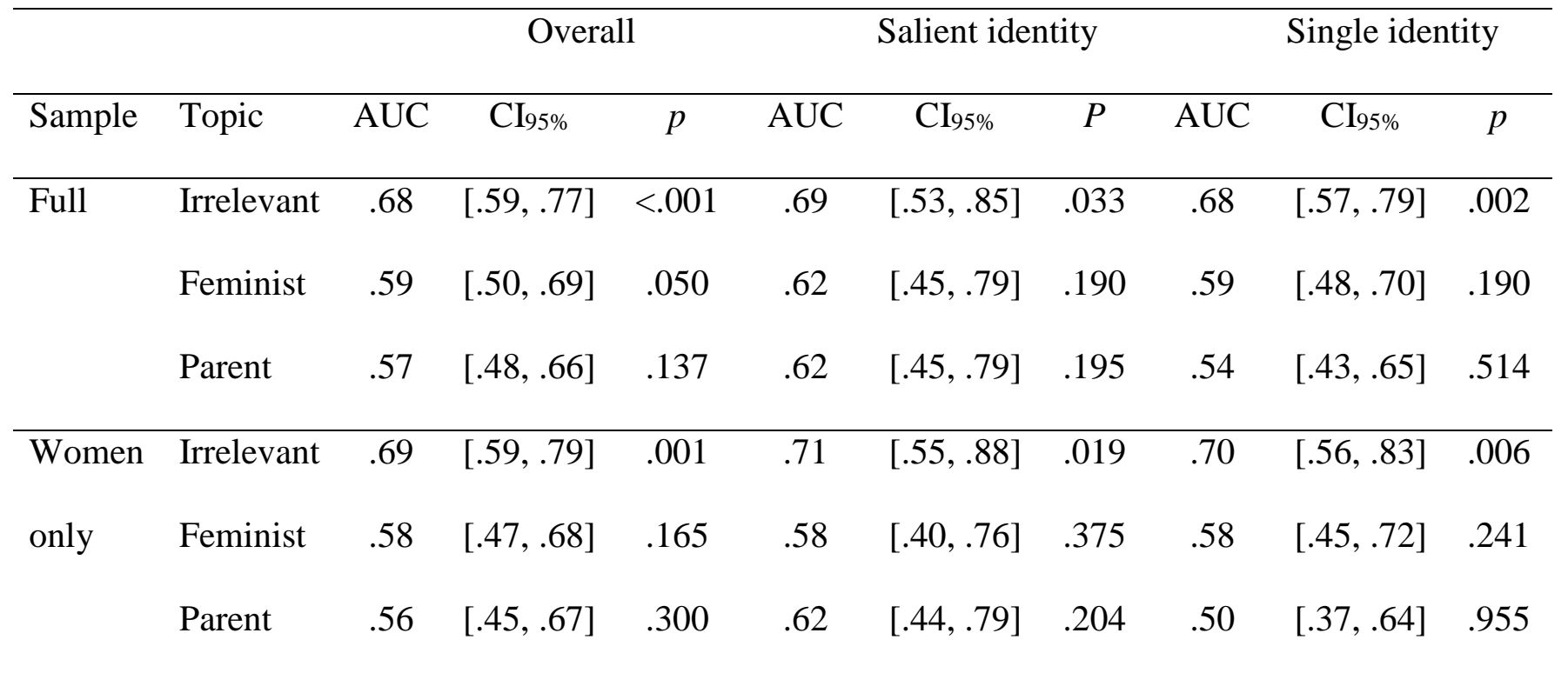

Note. AUC $=$ Area under curve $; \mathrm{CI}=$ confidence interval; Full sample: $N=152$ (overall)/ 42 43 (salient) / 109-110 (single); Women only sample: $N=115$ (overall)/ 40-41 (salient) / 7475 (single).

Results show that the model was most successful in distinguishing between the two social identities with an identity-irrelevant topic: The overall prediction accuracy was good and significantly above chance level, with $\mathrm{AUC}=.68$. A similar picture emerged when the data were analyzed separately for salient identity conditions and single identity conditions: For those holding both identities but with only one identity made salient (salient identity conditions), the prediction accuracy was good, with AUC $=.69$. Prediction accuracy for those with either a feminist or parent identity (single identity conditions) was similarly good, with $\mathrm{AUC}=.68$. The prediction rates showed comparable levels and remained significantly above 
chance level when the sample was restricted to women only (see lower half of Table 1 for results).

In contrast, topics more relevant for one identity or the other showed classifier performances at only chance level: For a topic relevant to feminists, prediction accuracy overall was AUC $=.59$. For a topic relevant to parents, overall prediction accuracy was AUC $=.57$. These results were equivalent for salient identity conditions and single identity conditions.

\section{Discussion}

A classifier based on only seven prototype-based stylistic features and trained on social media data was able to predict significantly above chance level which social identity an individual holds out of two overlapping group memberships in texts on an identity-irrelevant topic. Importantly, the classifier significantly predicted which social identity was salient during writing for those individuals who hold both social identities. Social identity remained a relevant source of variation even when topic, audience, and individual differences were experimentally controlled. These findings support our hypotheses that the stylistic pattern of social groups differs enough to allow for accurate classification of texts (H1), that prototypebased features are predictive (H2), and that the trained classifier will reliably distinguish between salient social identities where the individual holds both identities (H3).

However, the findings also show that predictive accuracy is topic-dependent, with an identity-irrelevant topic yielding the best predictive accuracy. One explanation why identityrelevant topics did not lead to a good predictive accuracy is that the topic itself may have made the relevant identity salient, thus over-writing our manipulation.

Remarkably, for the identity-irrelevant topic, the model was equally good or even slightly better at predicting whether a feminist or parent identity had been activated in the salient identity conditions compared with the single identity conditions. This finding suggests 
that being a member of a group does not exert constant influence on writing style; rather, it does so only when the identity is made psychologically salient. It therefore speaks to the context-dependent, dynamic nature of social identity as reflected in language style.

\section{General Discussion}

Previous automated analyses of social media data have provided evidence that stable individual characteristics can, to some extent, be accurately predicted. However, such approaches ignore the considerable intra-individual style shifts observed in sociolinguistic studies. More importantly, research in the SCT tradition provides ample evidence that group memberships do not exert a constant but instead dynamic, context-dependent influence on individual behavior (e.g., Haslam et al., 1999; Hogg \& Terry, 2000; Shih, Pittinsky \& Ambady, 1999). Our study contributes to this literature by showing salience shifts within the same individual rather than in a between-subjects design, and by using the behavior to predict the salient identity rather than the reverse. Specifically, in this paper, we provide first evidence that written text can be successfully employed to detect the salience of social identities, showing that individuals shift their language style in line with the group prototype. In Study 1, we demonstrate this effect in a within-subjects design using naturally occurring data, thereby extending the identity salience literature which employs experimental betweensubjects designs.

Our findings provide a proof-of-concept for computational tools to detect salient social identities as well as shifts between different identities within the same person based on the knowledge of the group prototype and a corpus of training data such as online forum entries. This gives social scientists the means to study effects of salient social identities at scale and using naturally occurring data, rather than having to rely on self-reports or observations. 
In sum, by combining an analysis of naturally occurring data and experimental data, we tested basic propositions of SCT (Turner et al., 1987). Firstly, we show that identities affect linguistic style in a dynamic rather than constant manner. Secondly, we demonstrate that behavior reflects the prototype of a relevant group when a social identity is salient. Thirdly, we provide evidence that salience of identity affects linguistic style irrespective of whether the audience is an ingroup (Study 1) or not (academics in Study 2), and when the topic is identity irrelevant (Study 2).

\section{Limitations and Future Directions}

One of the limitations to be considered in future research is that social identity is theorized to be highly context-dependent and relative in nature: The prototype of a group shifts with the comparative context (David \& Turner, 1999; Turner, Oakes, Haslam, \& McGarty, 1994). For instance, the prototype of a conservative political party is likely to be further to the right of the political spectrum when in debate with a liberal political party. When in debate with a more right-wing political party, the prototype is likely to shift momentarily towards the left (Haslam, 2004). Therefore, the linguistic cues to detect a social identity (e.g., feminist) are likely to depend on the relative comparison context (e.g., with parents). If compared with a different social identity (e.g., academic), particular linguistic cues (e.g., long words) should become less predictive. This limits the extent to which several social identities can be part of the same linguistic analysis simultaneously.

In our studies, prediction accuracy was remarkably good considering the small number of prototype-based features and the simple feature extraction we used. It also compares well with accuracies of AUC $=59-67 \%$ reported for personality prediction from social media data (Sumner, Byers, Boochever, \& Park, 2012). Future research using more sophisticated algorithms, such as neural networks, and open-vocabulary approaches (Schwartz et al., 2013) may boost accuracy further. 
In addition, future work should consider the possibility of deception, and whether knowledge of the group prototype is sufficient to successfully mislead automatic prediction of salient identities. Alternatively, the very act of deception may increase the salience of the actual identity which should undermine attempts at faking an outgroup identity. Intriguingly, the higher (rather than equal or lower) success rate of identifying demographic categories in fakers reported by Rashid and colleagues (2013) suggests the latter.

In the present research, we have sought to maximize statistical power in a number of ways. Firstly, in Study 1, we used a large naturally occurring dataset that allowed us to bootstrap our results. Small confidence intervals in Figure 1 show that 20 re-runs of our analysis on different sub-samples of the overall sample provide highly similar results, both for the training and test data. Secondly, by restricting our test data to an equal number of posts from users who had posted in both forums, we are also increasing power through a within-participant design. Thirdly, we are maximizing power by reducing the number of predictor variables in the prototype-based model while at the same time providing evidence that important information is not lost through a comparison with an all-features model. In Study 2, we calculated the sample size for power of .80 based on the findings from Study 1 . Here, it needs to be noted that we are not estimating predictors but instead are validating predictors from Study 1 in an experimentally controlled study. We have also sought to maximize power in Study 2 by using an explicit manipulation of salient identity in line with recommendations in the literature (see the appendix in Haslam, 2004, for a comparison of salience manipulation techniques). Although closed-vocabulary approaches such as LIWC that provide simple word counts are sometimes considered less predictive than openvocabulary approaches (Schwartz et al., 2013), LIWC has the advantage that it is a widely available software. Hence, our results can be easily replicated and our method can be used by others to produce classifiers for other social identities. 


\section{Potential Applications}

Most psychological research assesses social identity through self-report measures (Abdelal, Herrera, Johnston, \& McDermott, 2009). Occasionally, qualitative methods are used to analyze content in speech or writing, inferring social identities and how these are constructed in response to a dynamic social context such as national identity (Hopkins \& Reicher, 1996; Reicher, 1996). In everyday life, visible cues can be used to infer social identities such as age, gender, race, social class, and membership in organizations (e.g., through uniforms). However, most social identities are not easily visible and individuals may not always reveal or may even actively hide a social identity (Clair, Beatty, \& MacLean, 2005). Furthermore, visible cues tell us little about the psychological identity of the individual. Issues arise particularly with assigned categories (e.g. gender, race; Barreto \& Ellemers, 2003), but can equally be present in elected identities such as when a new recruit does not yet feel psychologically identified with the organization despite being a visible member of it (Mael \& Ashforth, 1995). Inferring social identity from behavior, particularly subtle forms of behavior, allows circumventing such problems posed by relying on visible cues and self-reports.

A further advantage of inferring social identities from evolving behavior is that the activation of a particular identity within a specific social context can be assessed as well as shifts between identities within the individual. Detecting shifts allows social scientists to learn more about the development and impact of social identities in natural social contexts in applied areas such as organizations, healthcare, marketing or security.

Such research, however, needs to assess potential ethical concerns. People often have legitimate reasons for hiding their identities. The notion of identifying people's 'true' allegiances is vulnerable for misuse. Although prediction accuracy is above chance level, our analyses show accuracy well below what would be necessary for a reliable individual-level 
diagnostic. We therefore impart on researchers a responsibility to consider the specific domains in which they employ methods of social identity salience detection.

Furthermore, researchers need to be aware when using naturally occurring data from online platforms that the data on which models are trained may introduce biases due to the particular demographic of users on that platform and within sub-forums (Nguyen et al., 2016). We used posts from a popular platform for UK parents, Mumsnet, to train our model. Even though this platform has over 10 million users, it does not reflect a representative sample of mothers/parents or feminists (Pedersen \& Smithson, 2013). For this reason, in Study 2, we explicitly recruited through various channels - different online forums and a paid survey platform - to ensure that our experimental validation study included participants who were in the majority not part of the initial forum on which we had trained our model. The finding that our Study 1 trained model is predictive of identity in Study 2 gives us confidence that our model can be applied to parents/feminists in the UK from a wider background than users on Mumsnet. However, given the nature of the study, we only had very few men in Study 2. Results show that findings were equivalent with men included and excluded but it is not possible to know with the current data whether the model works similarly well on men as on women. Here, it needs to be noted that the aim of the study was to test basic propositions of self-categorisation theory on dynamics in linguistic style and the influence of the prototype rather than creating a valid parent/feminist detection tool. Any future work that uses the methods described needs to ensure that models are supported experimentally on a diverse sample rather than based on naturally occurring data alone.

\section{Footnotes}

1 A random post per user and forum was used rather than all posts from users who had posted in both forums to avoid bias. That is, users who differ in personality or demographics (e.g., education) may post more in one forum than another. Using one post per forum for each user 
means that we can keep such differences between users constant, analogue to a withinparticipant design.

\section{References}

Abdelal, R., Herrera, Y. M., Johnston, A. I., \& McDermott, R. (2009). Measuring identity: A guide for social scientists. New York: Cambridge University Press.

Barreto, M., \& Ellemers, N. (2003). The effects of being categorised: The interplay between internal and external social identities. European Review of Social Psychology, 14, 139-170. http://dx.doi.org/10.1080/10463280340000045

Barreto, M., Spears, R., Ellemers, N., \& Shahinper, K. (2003). Who wants to know? The effect of audience on identity expression among minority group members. British Journal of Social Psychology, 42, 299-318. http://dx.doi.org/10.1348/014466603322127265

Bell, A. (1984). Language style as audience design. Language in Society, 13, 145-204. http://dx.doi.org/10.1017/S004740450001037X

Clair, J. A., Beatty, J. E., \& MacLean, T. L. (2005). Out of sight but not out of mind: Managing invisible social identities in the workplace. Academy of Management Review, 30, 78-95. http://dx.doi.org/10.5465/amr.2005.15281431

Coupland, N. (1980). Style-shifting in a Cardiff work-setting. Language in Society, 9, 1-12. http://dx.doi.org/10.1017/S0047404500007752

Coupland, N. (2007). Style: Language variation and identity. Cambridge, UK: Cambridge University Press.

David, B., \& Turner, J. C. (1999). Studies in self-categorization and minority conversion: The in-group minority in intragroup and intergroup contexts. British Journal of Social Psychology, 38, 115-134. http://dx.doi.org/10.1348/014466699164086 
Ennis, L. R. (2014). Intensive mothering: The cultural contradictions of modern motherhood. Bradford, Canada: Demeter Press.

Fast, L. A., \& Funder, D. C. (2008). Personality as manifest in word use: correlations with self-report, acquaintance report, and behavior. Journal of Personality and Social Psychology, 94, 334-346. http://dx.doi.org/10.1037/0022-3514.94.2.334

Franz, V. H., \& von Luxburg, U. (2015). No evidence for unconscious lie detection: A significant difference does not imply accurate classification. Psychological Science, 26, 1646-1648. http://dx.doi.org/10.1177/0956797615597333

Ganong, L. H., \& Coleman, M. (1995). The content of mother stereotypes. Sex Roles, 32, 495-512. http://dx.doi.org/10.1007/BF01544185

Giles, H., Taylor, D. M., \& Bourhis, R. (1973). Towards a theory of interpersonal accommodation through language: Some Canadian data. Language in Society, 2, $177-$ 192. http://dx.doi.org/10.1017/S0047404500000701

Goksuluk, D., Kormaz, S., Zararsiz, G., \& Karaagaoglu, A. E. (2016). easyROC: An interactive web-tool for ROC curve analysis using R language environment. The $R$ Journal, 8, 213-230. https://journal.r-project.org/archive/2016/RJ-2016042/index.html

Goldberg, A. E. (1995). Constructions: A construction grammar approach to argument structure: Chicago, IL: The University of Chicago Press.

Haslam, S. A. (2004). Social identity in organizations: The social identity approach. London, UK: Sage.

Haslam, S. A., Oakes, P. J., Reynolds, K. J., \& Turner, J. C. (1999). Social identity salience and the emergence of stereotype consensus. Personality and Social Psychology Bulletin, 25, 809-818. http://dx.doi.org/10.1177/0146167299025007004 
Haslam, S. A., Turner, J. C., Oakes, P. J., McGarty, C., \& Reynolds, K. J. (1997). The group as a basis for emergent stereotype consensus. European Review of Social Psychology, 8, 203-239. http://dx.doi.org/10.1080/14792779643000128

Hays, S. (1998). The cultural contradictions of motherhood. New Haven, CT: Yale University Press.

Hogg, M. A., \& Reid, S. A. (2006). Social identity, self-categorization, and the communication of group norms. Communication Theory, 16, 7-30. http://dx.doi.org/10.1111/j.1468-2885.2006.00003.x

Hogg, M. A., \& Terry, D. I. (2000). Social identity and self-categorization processes in organizational contexts. Academy of Management Review, 25, 121-140. http://dx.doi.org/10.5465/amr.2000.2791606

Hopkins, N., \& Reicher, S. (1996). The construction of social categories and processes of social change: Arguing about national identities (pp. 69-93). In G. M. Breakwell \& E. Lyons (Eds.), Changing European Identities: Social Psychological Analyses of Social Change. Oxford, UK: Butterworth-Heinemann.

Ishizuka, P. (2018). Social class, gender, and contemporary parenting standards in the United States: Evidence from a national survey experiment. Social Forces, soy107. https://doi.org/10.1093/sf/soy107

Klein, O., Spears, R., \& Reicher, S. (2007). Social identity performance: Extending the strategic side of SIDE. Personality and Social Psychology Review, 11, 28-45. http://dx.doi.org/10.1177/1088868306294588

Labov, W. (1968/2006). The social stratification of English in New York City. New York: Cambridge University Press. 
Laserna, C. M., Seih, Y. T., \& Pennebaker, J. W. (2014). Um... Who Like Says You Know: Filler Word Use as a Function of Age, Gender, and Personality. Journal of Language and Social Psychology, 33, 328-338. http://dx.doi.org/10.1177/0261927x14526993

Laver, M., Benoit, K., \& Garry, J. (2003). Extracting policy positions from political texts using words as data. American Political Science Review, 97, 311-331. http://dx.doi.org/10.1017/S0003055403000698

Le Page, R. B., Le Page, R. B., \& Tabouret-Keller, A. (1985). Acts of identity: Creole-based approaches to language and ethnicity. Cambridge, UK: Cambridge University Press.

Livingstone, A. G., Spears, R., Manstead, A. S., Bruder, M., \& Shepherd, L. (2011). We feel, therefore we are: emotion as a basis for self-categorization and social action. Emotion, 11, 754-767. http://dx.doi.org/10.1037/a0023223

Mael, F. A., \& Ashforth, B. E. (1995). Loyal from day one: Biodata, organizational identification, and turnover among newcomers. Personnel Psychology, 48, 309-333. http://dx.doi.org/10.1111/j.1744-6570.1995.tb01759.x

Mullen, B. (1991). Group composition, salience, and cognitive representations: The phenomenology of being in a group. Journal of Experimental Social Psychology, 27, 297-323. http://dx.doi.org/10.1016/0022-1031(91)90028-5

Myers-Scotton, C. (1993). Social Motivations for Codeswitching: Evidence from Africa. Oxford, UK: Clarendon Press.

Newman, M. L., Groom, C. J., Handelman, L. D., \& Pennebaker, J. W. (2008). Gender differences in language use: An analysis of 14,000 text samples. Discourse Processes, 45, 211-236. http://dx.doi.org/10.1080/01638530802073712

Nguyen, D., Doğruöz, A. S., Rosé, C. P., \& de Jong, F. (2016). Computational sociolinguistics: A survey. Computational Linguistics, 42, 537-593. https://dx.doi.org/10.1162/COLI_a_00258 
Park, G., Schwartz, H. A., Eichstaedt, J. C., Kern, M. L., Kosinski, M., Stillwell, D. J., . . Seligman, M. E. (2015). Automatic personality assessment through social media language. Journal of Personality and Social Psychology, 108, 934-952. http://dx.doi.org/10.1037/pspp0000020

Pedersen, S., \& Smithson, J. (2013). Mothers with attitude-How the Mumsnet parenting forum offers space for new forms of femininity to emerge online. Women's Studies International Forum, 38, 97-106. http://dx.doi.org/10.1016/j.wsif.2013.03.004

Pennebaker, J. W., Booth, R. J., \& Francis, M. E. (2007). Linguistic inquiry and word count: LIWC [Computer software]. Austin, TX: liwc.net.

Pennebaker, J.W., Chung, C., Ireland, M., Gonzales, A., \& Booth, R. (2007). The development and psychometric properties of LIWC2007. Retrieved from http://www.liwc.net/LIWC2007LanguageManual.pdf

Pennebaker, J. W., \& King, L. A. (1999). Linguistic styles: Language use as an individual difference. Journal of Personality and Social Psychology, 77, 1296-1312. http://dx.doi.org/10.1037/0022-3514.77.6.1296

Pennebaker, J. W., Mehl, M. R., \& Niederhoffer, K. G. (2003). Psychological aspects of natural language use: Our words, our selves. Annual Review of Psychology, 54, 547577. http://dx.doi.org/10.1146/annurev.psych.54.101601.145041

Rashid, A., Baron, A., Rayson, P., May-Chahal, C., Greenwood, P., \& Walkerdine, J. (2013). Who am I? Analyzing digital personas in cybercrime investigations. Computer, 46(4), 54-61. http://dx.doi.org/10.1109/MC.2013.68

Reicher, S. D. (1996). 'The Battle of Westminster': Developing the social identity model of crowd behavior in order to explain the initiation and development of collective conflict. European Journal of Social Psychology, 26, 115-134. http://dx.doi.org/10.1002/(SICI)1099-0992(199601) 
Ritter, R. S., Preston, J. L., \& Hernandez, I. (2014). Happy tweets: Christians are happier, more socially connected, and less analytical than atheists on Twitter. Social Psychological and Personality Science, 5, 243-249. http://dx.doi.org/10.1177/1948550613492345

Roy, R. E., Weibust, K. S., \& Miller, C. T. (2007). Effects of steretoypes about feminist on feminist self-identification. Psychology of Women Quarterly, 31, 146-156. https://doi.org/10.1111/j.1471-6402.2007.00348.x

Schmitz, C. (2012). LimeSurvey: An Open Source survey tool [Computer software]. Hamburg, Germany: Lime Survey Project. http://www.limesurvey.org

Schwartz, H. A., Eichstaedt, J. C., Kern, M. L., Dziurzynski, L., Ramones, S. M., Agrawal, M., . . . Ungar, L. H. (2013). Personality, gender, and age in the language of social media: The open-vocabulary approach. PLoS One, 8(9). http://dx.doi.org/10.1371/journal.pone.0073791

Shih, M., Pittinsky, T. L., \& Ambady, N. (1999). Stereotype susceptibility: Identity salience and shifts in quantitative performance. Psychological Science, 10, 80-83. https://doi.org/10.1111/1467-9280.00111

Sumner, C., Byers, A., Boochever, R., \& Park, G. J. (2012, December). Predicting dark triad personality traits from Twitter usage and a linguistic analysis of tweets. Paper presented at the 11th International Conference on Machine Learning and Applications, Boca Raton. http://dx.doi.org/10.1109/ICMLA.2012.218

Sylwester, K., \& Purver, M. (2015). Twitter language use reflects psychological differences between Democrats and Republicans. PLoS One, 10(9), e0137422. http://dx.doi.org/10.1371/journal.pone.0137422 
Tamburrini, N., Cinnirella, M., Jansen, V. A., \& Bryden, J. (2015). Twitter users change word usage according to conversation-partner social identity. Social Networks, 40, 8489. http://dx.doi.org/10.1016/j.socnet.2014.07.004

Tausczik, Y. R., \& Pennebaker, J. W. (2010). The Psychological Meaning of Words: LIWC and Computerized Text Analysis Methods. Journal of Language and Social Psychology, 29, 24-54. http://dx.doi.org/10.1177/0261927x09351676

Turner, J. C. (1991). Social influence. Buckingham, UK: Open University Press.

Turner, J. C., Hogg, M. A., Oakes, P. J., Reicher, S. D., \& Wetherell, M. S. (1987). Rediscovering the social group: A self-categorization theory. Cambridge, MA: Basil Blackwell.

Turner, J. C., Oakes, P. J., Haslam, S. A., \& McGarty, C. (1994). Self and collective: Cognition and social context. Personality and Social Psychology Bulletin, 20, 454463. http://dx.doi.org/10.1177/0146167294205002

Twenge, J. M., \& Zucker, A. N. (1999). What is a feminist? Evaluations and stereotypes in closed-and open-ended responses. Psychology of Women Quarterly, 23, 591-605. http://dx.doi.org/10.1111/j.1471-6402.1999.tb00383.x

Yarkoni, T. (2010). Personality in 100,000 words: A large-scale analysis of personality and word use among bloggers. Journal of Research in Personality, 44, 363-373. http://dx.doi.org/10.1111/j.1471-6402.1999.tb00383.x

Yopyk, D. J., \& Prentice, D. A. (2005). Am I an athlete or a student? Identity salience and stereotype threat in student-athletes. Basic and Applied Social Psychology, 27, 329336. http://dx.doi.org/10.1207/s15324834basp2704_5 